Res Pública Revista de Historia de las Ideas Políticas

ISSN: $1131-558 \mathrm{X}$

http://dx.doi.org/10.5209/rpub.66418

\title{
¿Qué fue de...? Breves apuntes sobre el capitalismo cognitivo y el modo de producción capitalista en el siglo XXI
}

\author{
Clara Navarro Ruiz*
}

Recibido: 13 de noviembre de 2019 / Aceptado: 13 de febrero de 2020

Resumen. En las siguientes líneas se aborda una de las corrientes dedicadas al estudio de las transformaciones sufridas por el modo de producción capitalista a partir de la introducción de las tecnologías de la información y el conocimiento (TICs), el capitalismo cognitivo. El estudio de sus especificidades y deficiencias argumentativas, que realizaremos con la ayuda de la posición de G. Caffentzis, nos permitirá, en la última sección de este artículo, realizar una breve consideración de los aspectos a abordar para un correcto análisis del capitalismo contemporáneo.

Palabras clave: Capitalismo cognitivo; TICs; Automatización; Fin del trabajo; Trabajo inmaterial.

\section{[en] Whatever Happened to...? Brief Considerations on Cognitive Capitalism and the Capitalist Mode of Production of the XXI Century}

\begin{abstract}
In these lines we tackle the contributions of cognitive capitalism, a stream of thought dedicated to analyze the transformations that Information and Communication Technologies (ICTs) have inflicted on the capitalism mode of production. Its specifies and theoretical shortcomings will be enlightened with the help of G. Caffentzis's considerations on these contributions. As a result of the preceding account, we will briefly explain some of the relevant aspects to consider within a fruitful analysis of contemporary capitalism.
\end{abstract}

Keywords: Cognitive Capitalism; ICTs; Automation; End of work; Immaterial work.

Sumario. 1. Saber y poder a la luz de las transformaciones del trabajo. 2. Peldaños invisibles, la clase del Capital y el mito de la cornucopia. 3. Mapa (mínimo) para la constitución de un análisis del capitalismo contemporáneo. 4. Bibliografía.

Cómo citar: Navarro Ruiz, C. (2020). ¿Qué fue de...? Breves apuntes sobre el capitalismo cognitivo y el modo de producción capitalista en el siglo XXI. Res Pública. Revista de Historia de las Ideas Políticas, 23(1), 97-108.

Comencemos por los datos contrastables. En el momento en que se escriben estas líneas, las cinco primeras empresas de mayor tamaño por su beneficio (según la Revista Fortune) son todas ellas, a excepción de Walmart, empresas dedicadas a la gestión y extracción de recursos energéticos. Al mismo tiempo, el índice Dow Jones contiene ya seis empresas dedicadas a la tecnología y/o comunicación entre sus primeras treinta referencias ordenadas por capitalización bursátil, dos de ellas (Microsoft y Apple) en el primer y segundo puesto, respectivamente. Por tanto, puede decirse con seguridad que el panorama del capitalismo está cambiando, si bien quizá no a la velocidad y del modo al que parece apuntar la insistencia del discurso político y mediático. Ya nos hemos acos- tumbrado al ruido de fondo que no cesa de explicar y alabar las bondades y peligros de la nueva economía asociada a las plataformas, start-ups, y en general, toda la revolución tecnológica a que asistimos desde la aparición de la computación ${ }^{2}$.

Sea como sea, el único modo de responder adecuadamente a lo que sugieren los datos mencionados es analizar la concordancia de los discursos teóricos que se han dedicado a tratar estos asuntos con la realidad actual. Precisamente esa es la tarea que nos hemos propuesto en las siguientes líneas, donde abordaremos una de las teorías que más pregnancia tuvieron durante un breve lapso de tiempo a comienzos de este siglo: el capitalismo cognitivo. Partiremos de la explicación de las tesis de dos de sus figuras más sobre-

Universidad Complutense de Madrid

claranavarro@ucm.es

l Los datos acerca de las empresas con mayor beneficio se han tomado de la lista realizada por y los índices bursátiles han sido tomados, respectivamente, las siguientes direcciones URL: [https://medium.com/technicity/worlds-100-largest-companies-by-revenue-in-2019-d6d53dd1851d] y [https://disfold.com/top-companies-us-dow/\#utc] [Visitado: 12-11-2019]. 
salientes, Carlo Vercellone y Yann Moulier-Boutang y, a continuación, expondremos las inconsistencias de sus argumentos. Dicho fin, que realizaremos gracias a las aportaciones de George Caffentzis, no nace de un espíritu obsesionado con la mera polémica. Antes bien, creemos que es solo de este modo como se puede identificar, in concreto, cuáles son aquellos aspectos que han de tenerse presentes al abordar la reflexión sobre un asunto concreto -en nuestro caso, la exposición de las especificidades del capitalismo contemporáneo-. Justo esto último es lo que tendremos ocasión de mencionar en nuestro último apartado, donde daremos cuenta de aquellos factores y cuestiones que no deben soslayarse, indicando asimismo algunos autores que están dedicando sus esfuerzos a arrojar luz sobre ellos.

\section{Saber y poder a la luz de las transformaciones del trabajo}

Puede decirse, de manera algo impropia, que "capitalismo cognitivo" es un término conceptual que agrupa algunas de las intervenciones críticas nacidas a raíz del surgimiento de la llamada new economy en la última década del siglo pasado. En ese momento estaba teniendo lugar la implementación decidida y manifiesta de las Tecnologías de la Información y la Comunicación (TICs) en el plano económico, que resultó en la proliferación de una gran cantidad de empresas que parecían prometer abrir una nueva era para las ganancias del capital. Como sabemos por la historia de la economía, la ilusión generada contribuyó a la formación de una burbuja especulativa que vivió sus años más robustos entre 1997 y 2001 y que, tras estallar, desembocó en la llamada crisis de las puntocom.

Desde luego, sería injusto decir que el trabajo de estos teóricos es la mera respuesta a un determinado statu quo. Sobre todo, teniendo en cuenta que sus contribuciones más notables han tenido lugar de manera posterior al lapso temporal mencionado ${ }^{2}$, y que, después de todo, lograron incidir de manera decidida en los círculos de reflexión más fuertemente ligados al activismo. Por tanto, no es en ningún caso un pensamiento urgente, tanto más, asumiendo que sus análisis están basados en planteamientos teóricos de trayectoria más amplia que han de remontarse a una

Nos referimos aquí, por ejemplo, a los textos reunidos bajo la revista Multitudes (heredera directa de Futur Antérieur), algunas de cuyas contribuciones fueron traducidas al castellano en el texto de O. Blondeau, N. Dyer Whiteford, C. Vercellone, A. Kyrou, A. Corsani, E. Rullani, Y. Moulier-Boutang y M. Lazzarato, Capitalismo cognitivo. Propiedad intelectual y creación colectiva, Madrid, Traficantes de Sueños, 2004. Aquí podemos observar que las diferentes contribuciones del capitalismo cognitivo no se limitan a los aspectos que consideramos en el cuerpo del texto, sino que abarcan consideraciones más generales acerca de la historia de la Economía Política como disciplina (cf., en el volumen citado el texto de A. Corsani, "Hacia una renovación de la economía política. Antiguas categorías e innovación tecnológica", pp. 89-98, así como el texto de E. Rullani, "Capitalismo cognitivo. ¿Un déjà vu?”, pp. 99-106) así como reflexiones más específicas acerca de la política de copyright y los nuevos tipos de relaciones que surgen a partir de la interconexión entre individuos a través de las telecomunicaciones. específica lectura de Marx. Nos referimos, por supuesto, a los planteamientos de Antonio Negri, que ya desde los años 70, venía realizando una consideración sobre ciertos aspectos de los Grundrisse de Marx, que culminaría posteriormente en sus reflexiones sobre trabajo inmaterial y capitalismo postfordista en Imperio, junto a Michael Hardt ${ }^{3}$.

El peso de dicho autor en el capitalismo cognitivo puede identificarse en su heterodoxa lectura de la teoría del valor, algunos de cuyos elementos fundamentales (capital, renta) se cargan de contingencia histórica. Asimismo, es visible en la tendencia de estos autores a comprender el capitalismo como un proceso de apropiación de la plusvalía y, por ende, como una específica manera de ejercer el poder, más que como un sistema de legaliformidad propia. Por último, de raigambre negriniana es, por descontado, la particular atención a las transformaciones del trabajo en el posfordismo. Tal como entienden, el trabajo vendría, cada vez más, a ser de carácter "inmaterial" y "cognitivo", como respuesta a las transformaciones tecnológicas. Consecuentemente, ambas propiedades dotarían a los/as trabajadores/as de nuevas capacidades para hacer posible la subversión de las relaciones instituidas.

En orden a circunscribirnos más específicamente al discurso de esta línea teórica, puede mencionarse a Yann Moulier Boutang, quien entiende que por capitalismo cognitivo ha de entenderse una nueva fase en el modelo de acumulación propio del capitalismo. En lugar de trabajo abstracto (es decir, trabajo muerto), ahora el principal objeto de acumulación es el conocimiento. Este, a causa de dicho cambio, se ha convertido en "la fuente básica de valor, así como en la localización principal del proceso de valorización"4. Esta sencilla definición se materializa en una serie de características de esta época, que pasamos a enumerar ${ }^{5}$.

Las contribuciones de Antonio Negri abarcan múltiples aspectos que no se circunscriben en absoluto a la teoría del valor. No obstante, para un acercamiento a sus planteamientos en este sentido, puede consultarse, amén del ya mencionado Imperio (M. Hardt y T. Negri, Imperio, Barcelona, Paidós, 2002, reflexión conjunta continuada en Multitud [Barcelona, Debate, 2004] y Commonwealth [Madrid, Akal 2011]), el artículo "Marx y el trabajo. El camino de la disutopía" (publicado en 1996 en Futur Anterieur, 35-36, pp. 180 y ss., recientemente traducido y publicado en castellano en F. Sierra Caballero (ed.), Teoría del valor, comunicación, territorio, Madrid, Akal, 2019, pp. 145-162). Por último, un acercamiento al concepto específico de "trabajo inmaterial" puede consultarse en su contribución junto a M. Lazzarato, "Trabajo inmaterial y subjetividad" (publicado en Futur Anteriéur, nº, 1991, disponible en la siguiente URL [http://www.rebelion.org/ docs/121986.pdf]) y sus lecciones sobre los Grundrisse impartidas en París en 1978 en Marx más allá de Marx (Madrid, Akal, 2011).

Y. Moulier-Boutang, Cognitive Capitalism, Cambridge, Polity Press, 2011, p. 57. (En adelante Cognitive Capitalism...). De este autor, cf., también "La crisis de la teoría del valor en la era del capitalismo cognitivo y las restricciones del antropoceno", en F. Sierra Caballero (ed.), op. cit., pp. 35-56. (En adelante "La crisis de la teoría..."), así como "Riqueza, renta, propiedad, libertad y renta en el capitalismo cognitivo", en O. Blondeau, N. Dyer Whiteford, C. Vercellone, A. Kyrou, A. Corsani, E. Rullani, Y. Moulier-Boutang y M. Lazzarato, op. cit., pp. 107-128.

En la siguiente enumeración, cf., ibidem, pp. 50-56. 
En primer lugar, en el capitalismo cognitivo son característicos i) la virtualización de la economía y la centralidad de los aspectos inmateriales y ii) la asimilación de la innovación creativa a través de los procesos de cooperación social entre individuos. De manera ulterior, la última peculiaridad mencionada hace que cada vez sea más difícil medir los factores que contribuyen al valor y a la producción de mercancías. Asimismo, en esta época se estaría dando un iii) cuestionamiento de la división del trabajo asumida por el fordismo, iv) así como una creciente complejidad de los mercados de mercancías, provocando la transformación de los procesos de producción (flexibilización, producción just in time). Ambas particularidades abren la posibilidad de verificar el declive del capitalismo industrial y de algunos de sus conceptos asociados, como el carácter individual del logro en el lugar de trabajo ${ }^{6}$. Esto último estaría relacionado con v) la creciente importancia de la interconexión entre sujetos (netware) para el proceso de acumulación, provocando que tengan, en general, una mayor relevancia los indicadores relativos a la productividad agregada. Dada esta situación, vi) las actividades que antes caían bajo el concepto de "trabajo", que cada vez se encuentran más fácilmente sustituidas por los algoritmos en constante transformación a través de la gestión del big data, pueden dividirse en dos grupos. De una parte, se dan una exigua serie de empleos creativos y transdisciplinarios, bien remunerados; por otro, una gran cantidad de empleos asistenciales, cuya importancia no encuentra reconocimiento en la asignación salarial obtenida, o sea, son altamente precarios ${ }^{7}$.

Por último, en lo que respecta al contexto de la economía, Moulier-Boutang reconoce la vii) centralidad en aumento de las externalidades sobre las economías, es decir, aquellos efectos colaterales o secundarios propiciados por una acción concreta del modo de producción capitalista. Estas son bien de carácter positivo (creación de puestos de trabajo en el sector servicios a causa de la introducción de una fábrica en un determinado lugar) o negativo (destrucción de la biodiversidad y entorno natural).

Esta caracterización general puede ser complementada por las contribuciones de Carlo Vercellone, quien de manera más continuada ha analizado la historia de las relaciones económicas recientes desde la perspectiva que aquí nos ocupa. Si atendemos a sus escritos $^{8}$, podemos ver que el concepto de "capitalis-

\footnotetext{
La anterior sentencia que realizamos comentando que el capitalismo cognitivo, si bien había de entenderse en un contexto de emergencia asociado a la aparición en escena de la new economy, no había de entenderse como mera respuesta a un determinado statu quo encuentra aquí su afirmación. Moulier Boutang no solo es crítico con esta nueva forma de acumulación, sino que explica que el fracaso del anteriormente novedoso modelo de la new economy se debe, precisamente, a las deficiencias propias del sistema capitalista, que es incapaz de tomar el control del ámbito de la información y el conocimiento con las escasas herramientas de las que dispone (Cf. "Riqueza...”, pp. 110-111).

Cf. F. Sierra Caballero, "La crisis de la teoría...", op. cit., p. 42-44.

8 Aquí nos referiremos a su texto "Crisis del valor y cambios en la relación capital/trabajo” en F. Sierra Caballero (ed.), op. cit., pp. 213-
}

mo cognitivo" también implica una serie de cambios metodológicos en el estudio del capitalismo.

De manera contraria a lo que parecería afirmar un burdo determinismo tecnológico que hubiera fijado su atención en las nuevas tecnologías, de entrada se debe tener presente que el capitalismo cognitivo asume como presupuesto que el conocimiento y la educación son los medios principales de expresión y creación del trabajo9 ${ }^{9}$. En la nueva etapa de acumulación del sistema capitalista, su significado habría incrementado su importancia y desplazado su posición funcional dentro del sistema productivo. Esta circunstancia ha dado lugar a que la antigua oposición entre el trabajo vivo - actividad exclusiva de la fuerza de trabajo- y el trabajo muerto -cristalizado en la maquinaria que conforma el capital constante e inscrito en el saber de la sociedad, que conforma así un estándar de productividad determinado- haya dado paso a un nuevo antagonismo potencialmente conflictivo, capaz de trastocar la relación capital/trabajo: el existente entre el conocimiento muerto del capital y el conocimiento vivo del trabajo. Como ya se ha expresado en cierto modo con Boutang, este conocimiento vivo ha de comprenderse como un conocimiento social, común y colectivo, más cercano a la metáfora de la labor de polinización de las abejas. Más bien, se trata de una "intelectualidad difusa" que "es el punto de partida de un proceso de reapropiación colectiva de las potencias intelectuales de la producción, que constituye una condición previa necesaria para la superación de la división capitalista-industrial del trabajo"10.

Tal como puede leerse, dicha intelectualidad, más allá de su potencial capacidad subversiva por la posibilidad de reapropiación que habita en ella, es una primera condición para la superación de la división industrial del trabajo, algunos de cuyos síntomas ya son visibles. En primer lugar, y dentro de la línea de lectura marxiana de Negri, Vercellone afirma que la reaparición del aspecto cognitivo del trabajo hace que la ley del valor fundada en el tiempo de trabajo haya entrado en crisis. Esta aseveración implica algunas asunciones que deben explicitarse.

Según Vercellone, en el capitalismo industrial, la medida, catalizador y fin del desarrollo de la riqueza social se encuentra en la producción en masa de bienes, que han de reducir su valor individual constantemente. Ello se ha hecho posible a través de una específica configuración sociohistórica de la ley de la plusvalía capitalista, esto es, por una forma concreta de las relaciones de poder que permiten la explotación

242 (en adelante "Crisis del valor..."), así como "From formal subsumption to general intellect: elements for a Marxist reading of the thesis of cognitive capitalism", Historical Materialism, 17, 2007, pp. 13-36 DOI: [https://doi.org/10.1163/156920607X171681] (en adelante "From formal..."), y "Crisis del valor y devenir renta de la ganancia. Apuntes sobre la crisis sistémica del capitalismo cognitivo", en A. Fumagalli, S. Lucarelli, C. Marazzi, S. Mezzadra, A. Negri y C. Vercellone, La gran crisis de la economía global. Mercados financieros, luchas sociales y nuevos escenarios políticos, Madrid, Traficantes de Sueños, 2009, (en adelante “Apuntes...”).

Cf. en estas afirmaciones y las siguientes argumentaciones, C. Vercellone, "From formal...", op. cit., pp. 33-34.

10 C. Vercellone "Crisis del valor...", op. cit., p. 217. 
de unos individuos sobre otros y marcan los límites del antagonismo entre los diversos grupos sociales. La que aquí nos concierne es la de la ley del valor de tiempo de trabajo. Surgida tras la Segunda Revolución Industrial ${ }^{11}$, se materializa en una disciplina y un proceso de abstracción del contenido concreto del trabajo. Por esta fue como se hizo, primero del tiempo del reloj y luego del tiempo del cronómetro, los instrumentos por antonomasia para medir y cuantificar el valor económico del trabajo. Gracias a ellos, se podían realizar prescripciones sobre el modo en que habían de llevarse a término las tareas, de modo que pudiera aumentarse la eficiencia en su efectuación, mejorando la productividad ${ }^{12}$. Por supuesto, estos instrumentos de medida y prescripción se encuentran en el marco de la vigencia de la ley reguladora del tiempo de trabajo socialmente necesario, que garantiza ex post - una vez ejecutada la realización de las mercancías por medio de su intercambio efectivo en el mercado- las relaciones de competencia entre los productores privados, enfrentados entre sí.

De esta manera, el universo fordista por antonomasia, cuyo orden habría hecho las delicias de cualquier teórico funcionalista, se encontraba en su tipo ideal conformada por una estructura tripartita ${ }^{13}$. En primer lugar, i) la empresa, con un dirigente de tipo galbraithiano a la cabeza, estaba constituida por una organización científica del trabajo dividida bajo el criterio de la oposición entre trabajo intelectual/trabajo manual. El primer tipo de trabajo quedaba reservado a los llamados "cuellos blancos", que concentraban el esfuerzo innovador en la sección de $\mathrm{I}+\mathrm{D}$, mientras que al resto de trabajadores les aguardaba la realización del trabajo manual, que, descompuesto en sus elementos más simples, era necesariamente monótono y repetitivo. Además, la empresa fordista estaba directamente implicada en la creación de plusvalía, pues era tanto el elemento interno como la conditio sine qua non para la correcta dirección y organización del trabajo. Esta situación permite afirmar que la ganancia era el motor principal de desarrollo social ${ }^{14}$.

11 En realidad, Carlo Vercellone no afirma de manera expresa que esta configuración de las relaciones entre capital y trabajo hayan surgido tras la Segunda Revolución Industrial, se limita a afirmar que habrían surgido "tras la Revolución Industrial". No obstante, la caracterización que Vercellone realiza y un rápido acercamiento a la historia de las revoluciones industriales, nos permite afinar, casi con total seguridad, que la época específica a la que se refiere el autor es la de la Segunda Revolución Industrial, que tuvo lugar a finales del siglo XIX. Esta está asociada al paso de la industria ligera a la pesada, que pusieron en el centro de la economía al acero, al hierro y al carbón como materiales primas y fuentes de energía fundamentales y que impulsó la creación de una red de ferrocarril. En esta época surgieron textos como Los principios de economía de Marshall (1890), cuyas teorías para el aumento de la productividad de los trabajadores pasaban por la imposición de una jornada laboral menos extensa y una mejora generalizada de las condiciones de vida de la clase obrera, incluyendo a todos los miembros de la familia. Se trata de la época de aparición del modelo de familia nuclear burguesa con el hombre "ganapán" a la cabeza. Es asimismo de la era en que hicieron aparición las nuevas metodologías de trabajo de Taylor y Ford (Cf. S. Federici, El patriarcado del salario. Críticas feministas al marxismo. Madrid, Traficantes de Sueños, 2018, pp. 70 ss.).

12 C. Vercellone, "Crisis del valor...", op. cit., p. 219.

13 En la siguiente argumentación, cf., ibidem, pp. 223-224.

14 Ibidem, p. 226
Por su parte, ii) en la sociedad regía una clara distinción de los tiempos vitales: el de reproducción, inextricablemente ligado a la prestación gratuita del trabajo de cuidados y doméstico por parte de las mujeres, el de consumo $\mathrm{y}$, por último, el de trabajo, que se entendía dividido en las etapas educativa, profesional y de jubilación. Finalmente, iii) en lo que respecta al contrato social entre capital y trabajo, en la sociedad fordista existía una renuncia explícita de los/as trabajadores a cualquier tipo de reivindicación del producto de su trabajo a cambio de su salario.

A pesar de la importancia que esta configuración de relaciones pueda tener para el presente, Vercellone afirma que la ley de la plusvalía, aquella "que hace del plustrabajo el origen del beneficio y las rentas"15 es sí, el principio que estructura el funcionamiento del capitalismo, pero, también, algo distinto a y autónomo respecto de la forma histórica que acabamos de detallar. Tanto es así, que puede entrar en contradicción con ella, tal como, efectivamente, estaría ocurriendo en la época del capitalismo cognitivo, en varios ámbitos.

De entrada, cuestionado la norma del tiempo de trabajo como medida del valor. En las unidades productivas empresariales la creación de valor tiende ahora a desvincularse respecto de la "unidad de lugar y tiempo", es decir, que la "producción de riqueza y conocimiento se crea cada vez más por encima del sistema de empresas y de la esfera mercantil". Esto implica, a su vez, que el criterio del reparto del trabajo pertenezca al ámbito cognitivo, lo que significa que la eficacia pasa a depender de la polivalencia y conocimiento de la fuerza de trabajo ${ }^{16}$. De igual modo, los/as trabajadores comienzan a cuestionar los acuerdos que sostenían al sistema fordista: el salario ya no es suficiente para impedir las reivindicaciones de la fuerza de trabajo respecto de la propiedad del producto de su labor y, además, el tiempo libre ha abandonado su anterior función para el recobro del aliento. El tiempo forma ahora un continuum de constantes "actividades de formación, autoformación, trabajo voluntario, formación de comunidad de codivisión y producción de saberes, que atraviesan las distintas actividades humanas"17.

Por otro lado, es posible observar modificaciones al nivel de la estructura del sistema de acumulación, en lo referente a las herramientas utilizadas para la captación de la plusvalía. El teórico francés afirma que actualmente puede constatarse un retorno de la importancia de la renta en detrimento de la anterior lógica de la ganancia, cuya imbricación directa con el modelo de la empresa se ha explicado anteriormente. Las fronteras entre ganancia y renta se encuentran cada vez más cuestionadas, pues existe una creciente dependencia de la competitividad de las empresas respecto de su sola capacidad de identificar y apropiarse de sobreproducciones cognitivas de un territorio. A su vez, eso significa que el beneficio es cada vez más producto de la sola relación de distribución, dado que el capital absorbe una plusvalía externa en cuya organización productiva no ha contribuido en

\footnotetext{
Ibidem, p. 220.

$16 \quad$ Ibidem, p. 230.

17 Ibidem, pp. 233-234.
} 
absoluto $^{18}$. Bajo esta lógica subyace una importante consecuencia de la ruptura de la ley del valor del tiempo de trabajo $^{19}$. La creciente importancia del eje del saber en la acumulación de capital ha provocado que el tiempo de trabajo inmediato exigido para la producción mengüe, por lo que su valor monetario se ha reducido, y con ello, los beneficios del capital. Ante esta situación, para salvaguardar la legaliformidad del sistema del valor de cambio y, en definitiva, la vigencia de la ley de beneficio capitalista, el capital ha comenzado a utilizar mecanismos ligados a la renta para enrarecer la oferta. Por todo ello, es la dinámica rentista la que, según el autor francés, estaría hoy por hoy sosteniendo al sistema socioeconómico capitalista.

Por descontado, esta serie de transformaciones conllevan importantes consecuencias a nivel social. El capital inmaterial, habiendo superado en la actualidad la parte de capital material en el conjunto del stock real del capital, ha entrado en conflicto con la anterior categoría de "capital constante", que, como se ha dicho, está representado por el trabajo muerto cristalizado en las máquinas. Bajo el signo de los nuevos tiempos, el nuevo capital fijo está comenzando a ser la inteligencia colectiva, que no se puede medir de manera objetiva. Esto implica que el valor sea, cada vez en mayor medida, "la expresión subjetiva de la anticipación de beneficios futuros efectuada por los mercados financieros" 20 , lo que significa, a su vez, que el capitalismo no depende únicamente de los mecanismos de la renta, sino cada vez más de los mecanismos financieros. En palabras de Vercellone, efectivamente, en la actualidad

...la medida del capital y los cimientos del poder en la sociedad dependen de una convención social que encuentra su principal instancia material en el poder y la "violencia de la moneda". Sin embargo, el valor "bursátil" de dicho capital es básicamente ficticio. Este se fundamenta en una lógica autorreferencial de la finanza que estaba destinada a estallar, amenazando al sistema global del crédito y al conjunto de la economía de una crisis sistémica ${ }^{21}$.

Como puede verse, la descripción del capitalismo contemporáneo por parte de los teóricos de la corriente del capitalismo cognitivo nos lo presenta como un conjunto inherentemente inestable. Carente de una legaliformidad interna que permita su estabilidad -algo que en el pasado ofrecía la ley del tiempo de trabajo asociado al valor- ha de apoyarse en los mecanismos de la renta, la violencia de la moneda y los instrumentos financieros para poder seguir atando a su yugo los destinos de la fuerza de trabajo. Subvertir la correlación fuerzas existente depende de la potencia ínsita en la capacidad de cooperación de los trabajadores cognitivos, cuya relación con el proceso de trabajo impide que pueda contentarse con el salario como única contraprestación a su labor. Una labor que, además, cada vez menos puede calificarse como algo "individual" o "propia", sino que responde, más bien, a las lógicas de lo común.

\footnotetext{
Ibidem, pp. 236-237.

C. Vercellone, "Apuntes...", op. cit., p. 80-81.

C. Vercellone, "Crisis del valor...", op. cit., p. 221.

Ibidem, p. 221
}

A pesar de que algunas de sus aportaciones puedan resultar sugerentes, cabe preguntarse hasta qué punto podemos considerar consistentes las afirmaciones de esta corriente, es decir, si logran explicar adecuadamente los fenómenos visibles del capitalismo y si están rigurosamente fundamentadas en las tesis de Marx, autor que reclaman para sí. Las siguientes líneas están dedicadas a dar respuesta a estas inquietudes. Para ello, haremos uso de los argumentos de otro autor, George Caffentzis, que ya emprendió la labor de crítica que ahora vamos a continuar.

\section{Peldaños invisibles, la clase del Capital y el mito de la cornucopia}

Comenzaremos afirmando que una revisión sistemática de los errores del capitalismo cognitivo excede con mucho a la extensión a la que nos debemos en este artículo. Ello se debe, sin duda alguna, a la característica más sobresaliente de su teoría: el carácter fuertemente elusivo de los conceptos que utiliza, una ambigüedad y carácter lábil que, como sabemos, son enemigos íntimos de la comprensión filosófica. En el caso que nos ocupa, la equivocidad de los conceptos utilizados hace que el discurso carezca de una estructura sólida, creando aparentes oposiciones donde no hay relación alguna entre conceptos, e invisibilizando elementos que habrían de ser introducidos en la discusión.

Como podemos imaginarnos, esta confusión se refleja especialmente en su noción de "conocimiento" y en el epíteto "cognitivo". Tal como denuncia Caffentzis en sus textos ${ }^{22}$, puesto que este último término se está usando para denotar la novedad histórica de nuestro modo de acumulación actual, habría de haberse especificado con claridad a qué se está refiriendo dicha noción. Lo que es más: los autores habrían de haberse enfrentado a ciertos dilemas epistemológicos, como si la verdad o falsedad de un conocimiento/hecho determinado tiene algún tipo de influencia en su posible validez funcional para un sistema determinado (en este caso, el capitalismo).

Que los teóricos del capitalismo cognitivo no hayan tenido en cuenta estos problemas denota que desconocen que su verdadero objeto de estudio no es el conocimiento, ni la educación, sino antes bien, la capacidad de mercantilización y uso productivo de "los productos intelectuales, computacionales, mentales y digitales [...] del trabajo" y "no su estatus en tanto que conocimiento o cognición" 23 . El uso confuso que realizan de este concepto, que se encuentra en ellos a medio camino entre su status como factor de producción (y de valorización), como elemento subjetivo del trabajador y como característica social, es lo que permite que, ora pueda pri-

\footnotetext{
22 Cf. G. Caffentzis, "A critique of cognitive capitalism", en G. Caffentzis, In letters of Blood and Fire. Work, machines and the Crisis of Capitalism, Oakland, PM Press, 2013, pp. 95-126, pp. 108 (en adelante "A critique..."). En las siguientes líneas, nos referiremos asimismo a su artículo "The end of work or the renaissance of slavery? A critique of Rifkin and Negri", en G. Caffentzis, op. cit., pp. 6681 (en adelante "The end..."), así como a "Immesurable Value? An Essay on Marx's Legacy", The Commoner, n⿳10, primavera-verano, 2005, pp. 87-114. (en adelante "Immesurable Value?...").

23 Ibidem, p. 108.
} 
vilegiarse como un elemento de posible subversión de las relaciones de plusvalía; ora sea el elemento decisivo para la acumulación del capital, ora el factor que permita a los trabajadores de una empresa reapropiarse del producto de su trabajo. Que el cui bono de un elemento sirva simultáneamente a intereses contradictorios, implica que su significado ha de ser necesariamente amplio y/o abierto, pues en caso contrario no suscitaría multitud de interpretaciones. Algo que, evidentemente, es perfectamente válido en el caso de un símbolo o un reclamo publicitario, pero no en el caso del análisis y juicio de cualquier asunto.

Sea como sea, esta pequeña crítica introductoria es suficiente para adivinar el amplio alcance de la reestructuración discursiva necesaria para ordenar en sus justos quicios los elementos del capitalismo cognitivo. A causa de ello, en las siguientes líneas, creemos que puede ser más útil ceñirnos a discutir únicamente algunos de los aspectos más relevantes, sin pretensión de abarcar todos sus argumentos. Más concretamente, dedicaremos las siguientes líneas a tratar su concepción de la renta, su concepción de la creación de valor - para lo que tendremos que hacer referencia al uso de maquinaria-y su concepción del conocimiento como un factor productivo.

Comencemos por la noción de renta. Tal como hemos visto líneas más arriba, Vercellone argumentaba que los cambios en la estructura del sistema capitalista habían provocado que su progreso y desarrollo ya no dependiera de la dinámica de la ganancia (asociada a la producción en masa), sino a mecanismos propios de la renta, que extraen la plusvalía de las sobreproducciones cognitivas de un territorio sin haber ejercido influencia alguna en su producción. Para Vercellone, por tanto, la posible influencia en la producción de los capitalistas su labor directiva en el proceso de producción- es un factor relevante para la formación de la tasa de ganancia y/o plusvalía. Esta sola afirmación, sin poseer demasiada longitud, es suficiente para demostrar con claridad que Vercellone desconoce la obra marxista, pues oblitera los detalles más básicos que componen la formación de la tasa de ganancia. Expliquemos en qué sentido.

Dicho de manera sencilla, y tal como Caffentzis nos recuerda ${ }^{24}$, Karl Marx, en el capítulo noveno y siguientes del Tercer Tomo de El Capital, muestra que la formación de la tasa de ganancia implica la equiparación de todas las tasas de plusvalor de los diferentes ramos industriales de un país determinado (no olvidemos que la crítica de la economía política lo es siempre de un territorio nacional). Es decir, que la labor directiva de los capitalistas en la producción no tiene la importancia que Vercellone le otorga, y que la teoría del filósofo de Tréveris apunta en otra dirección.

En efecto, Marx, en los capítulos citados, explica cómo la tasa general de ganancia se conforma siempre a través del promedio de las diferentes tasas de ganancia para una cantidad determinada en un plazo de tiempo estipulado. Comenta igualmente que esta se encuentra condicionada tanto por 1) la composición orgánica de los diferentes capitales así como por 2) la distribución del capital social total en las diferentes áreas, esto es,

${ }^{24}$ Cf., ibidem, pp. 119-120. su participación cuantitativa. Siendo esto asín ${ }^{25}$, el beneficio de una empresa nunca se puede entender resultado de su exclusivo esfuerzo, sino que se ha de considerar como un factor variable, dependiente de una multitud de circunstancias. En síntesis, es resultado de un proceso de transformación, que parte del capital total social y lo redistribuye siguiendo la siguiente regla: siendo $c$ el capital constante, $v$ el capital variable en una rama determinada de la industria, y $R$ la tasa media de beneficio de todas las industrias, el beneficio sería calculable a partir de la fórmula $(c+v) R$, con la condición de que exista el libre movimiento de trabajo y capital. Que el resultado de esta sencilla fórmula tenga que tener en cuenta el promedio de la totalidad es lo que constituye, por su parte, la condición para la creación de una clase común de los capitalistas $^{26}$.

Por tanto, queda claro que el núcleo del argumento del capitalismo cognitivo, que afirma que la estructura de la acumulación capitalista se modifica a tenor de la voluntad de explotación de los dirigentes industriales, es claramente incorrecta. Se trata de una conclusión a la que podrían haber llegado por sí mismos, sin tanto rodeo teórico, si hubieran atendido asimismo a uno de los fragmentos más célebres de El Capital, el de la estructura trinitaria, formulada al comienzo de la última sección de su tercer tomo ${ }^{27}$. El fragmento de la estructura trinitaria presenta a la sociedad capitalista como el reino del fetiche consumado, donde nada aparenta ser lo que es en realidad: ni el trabajo asalariado, ni la propiedad del suelo, ni el capital pueden ser considerados (tal como la conciencia espontánea capitalista quisiera creer) condiciones materiales de la producción. Son resultado de la específica producción capitalista, basada en la constante acumulación de valor. Partiendo de esa necesidad de acumular valor, los diferentes ciclos de realización de las mercancías y acumulación de capital, considerados en su globalidad, conforman la masa de plusvalor social global, el todo a partir del que los diferentes actores de la economía obtienen sus réditos. En el caso del capital, esto incluye tanto a las industrias con alta y baja compo-

25 Cf., en lo siguiente ibidem, p. 119.

26 Cf. K. Marx, MEW 25: 170-172. Traducción española en El Capital, tomo tercero, volumen 6, pp. 203-205. Madrid, Siglo XXI, 2009 [1981]. La afirmación de esta clase común de los capitalistas se expresa, entre otros lugares, en el siguiente párrafo: “...los capitalistas de las diversas esferas de la producción, al vender sus mercancías, retiran los valores de capital consumidos en la producción de esas mercancías, no rescatan el plusvalor, y por ende la ganancia, producido en su propia esfera durante la producción de esas mercancías, sino solo la cantidad de plusvalor, y por ende de ganancia, que corresponde a cada parte alícuota del capital global por distribución uniforme del plusvalor global o de la ganancia global producida en un plusvalor global o de la ganancia global producida en un lapso dado por el capital global de la sociedad en el conjunto de todas las esferas de la producción. [...] En este aspecto, en tanto se toma en consideración la ganancia, los diversos capitalistas se conducen como meros accionistas de una sociedad por acciones, en la cual las participaciones en las ganancias se distribuyen equitativamente por cada 100, y que por ello solo se diferencias para los diversos capitalistas según la magnitud de capital invertido por cada cual en la empresa global,según su participación relativa en la empresa global, según el número de acciones que posea" (Marx, MEW 25, 168. Traducción española op. cit., p. 200).

27 K. Marx, MEW 25, 822-839. Traducción española op. cit., pp. 1037 1058. 
sición orgánica, esto es, aquellas en cuya producción se utiliza una extensa proporción de capital constante (alta composición orgánica, el caso de las ramas industriales acompañadas de una fuerte implementación de elementos "cognitivos") o una mayor proporción de capital variable (baja composición orgánica, industrias donde el factor productivo fundamental es la fuerza de trabajo).

Esto último nos lleva a un asunto que no carece de interés: tal como es sabido, el aumento de la productividad, producida por la mejora en eficiencia inducida por la introducción de tecnología, es el factor principal en la caída tendencial de la tasa de ganancia. Si el beneficio de las industrias con una mayor composición orgánica dependiera exclusivamente de sí mismas, lo más probable es que no alcanzarían siquiera el beneficio medio, imposibilitando su sola existencia. Teniendo en cuenta que el beneficio de los capitalistas se ha de considerar respecto de la totalidad del valor creado, puede decirse que estas ramas/empresas de la industria se benefician respecto del plusvalor creado por las industrias con una menor composición orgánica, pudiendo así alcanzar, cuanto menos, el ya nombrado beneficio medio ${ }^{28}$.

A pesar del alto grado de abstracción de lo que se está comentando, esta cuestión es importante, particularmente, por las consecuencias efectivas que tiene para la realidad social. Habida cuenta de lo discutido, el único modo para compensar la tendencia a la caída de la tasa de ganancia de las empresas de alta composición orgánica es la introducción de industrias que tengan una menor composición de capital y, en general, la implementación todos aquellos elementos que puedan actuar como "causas contrarrestantes". Por decirlo de algún modo, el extraccionismo violento en los países considerados exportadores de materias primas, las condiciones laborales en las zonas de procesamiento de exportaciones, así como el aumento de la masa global de trabajo precario (usualmente también migrante), es la otra cara de la moneda de las industrias cogniti$\operatorname{vas}^{29}$. Caffentzis lo expresa muy bien en el siguiente fragmento dedicado a Negri:

Negri niega imperiosamente "las leyes sociales y económicas que gobiernan la utilización de la fuerza de trabajo entre los diferentes sectores de la producción social" y niega que "el tiempo de trabajo es crucial para los procesos capitalistas de valorización”. Pero el capital y los capitalistas todavía están sinceramente interesados en ambos. Por eso hay esa inclinación a mandar capital a las áreas con menores salarios y por lo que existe tanta resistencia a la reducción de la jornada laboral. [...] ¿Por qué el Capital se

28 Cf. G. Caffentzis, "The end of work...", op. cit., pp. 78-79; "A critique...", op. cit., pp. 121-122.

29 Las "causas contrarrestantes" a la caída tendencial de la tasa de ganancia pueden dividirse, según Marx (MEW 25, 242-250, traducción española op. cit., 297-308) en las siguientes estrategias: elevación del grado de explotación del trabajo, reducción del salario por debajo de su valor, abaratamiento de los elementos del capital constante, la sobrepoblación relativa, el comercio exterior y el aumento del capital en acciones. Asimismo, para una pequeña imagen de las condiciones de trabajo, particularmente femenino, en las zonas de procesamiento de exportaciones $\mathrm{cf}$. T. Bhattacharya, "Explaining gender violence in the neoliberal era", International Socialist Review, 91, disponible en la siguiente URL: [https://isreview.org/issue/91/explaining-genderviolence-neoliberal-era]. preocupa de la titularidad de las tierras comunales en África, por ejemplo, si la verdadera fuente de la productividad se encuentra en los cyborgs del planeta? Una respuesta es simplemente que estas fábricas, tierras y burdeles en el tercer mundo son escenarios de las "causas contrarrestantes" a la tendencia de la caída de la tasa de ganancia. Aumentan el monto total de plustrabajo, ayudan a disminuir los salarios, abaratan los elementos del capital constante, y expanden de manera tremenda el mercado de trabajo y hacen posible el desarrollo de industrias high-tech que emplean de manera directa solo unos pocos trabajadores cognitivos o cyborgs ${ }^{30}$.

Tras esto, es momento de pasar a desentrañar más específicamente las deficiencias de la consideración del capitalismo cognitivo acerca del papel del conocimiento como factor de producción. Al comienzo de esta sección ya se ha puesto de manifiesto el carácter profundamente elusivo que este término posee, que provoca que adquiera, cuanto menos, tres significados que se confunden en su argumentación: factor de la producción decisivo para la valorización, elemento perteneciente a la subjetividad del trabajador y factor social. En todos los casos, los teóricos del capitalismo cognitivo definen al conocimiento como algo inconmensurable, factor que lo dota de su capacidad desestabilizadora.

Para garantizar el orden en la discusión, podemos hacemos uso nuevamente de la argumentación caffenztia$\mathrm{na}^{31}$. El autor estadounidense observa que la caracterización del trabajo cognitivo de estos autores es criticable por tres motivos. En primer lugar, se ha de partir del sencillo hecho de que, que haya uso de trabajo vivo en industrias asociadas a los recursos cognitivos, no quiere decir que todo el trabajo vivo que allí se utilice sea, bien trabajo cognitivo, bien trabajo productivo de conocimiento. Importa únicamente que este sea capaz de generar un valor de cambio, de manera autónoma a si tiene contenido epistémico. Para tal fin, tan solo debe satisfacer una necesidad humana, sin importar cuál sea su origen o el grado de creatividad que lo acompaña. Por tanto, el calificativo "cognitivo" no tiene, en este particular aspecto, ninguna importancia particular. Por contra, atendiendo a las capacidades subversivas que le otorgan, parece que los teóricos del capitalismo cognitivo utilizaran los términos "conocimiento" y "cognitivo" como si asimismo significaran de manera inmediata "autonomía" y "creatividad". Ahora bien, ni siquiera especifican si con dicho término se están refiriendo, en igual medida, tanto al ingeniero informático inmerso en un proyecto para la automatización y mejora de la eficiencia de la fabricación de turbinas de aviones o al community manager de un joven periódico que, bajo un régimen de prácticas no remuneradas, regala sus esfuerzos a buscar titulares que generen el deseado clickbait para su diario. Actividades muy diferentes a las que no podemos estar seguros si estarían dispuestos a calificar, en idéntico modo, como "autonómas" y "creativas". La mejora terminológica parece obligada.

\footnotetext{
G. Caffentzis, "The end of work...", op. cit., pp. 78-79.

31 Cf. G. Caffentzis, “A critique..., op. cit., pp. 112-113.
} 
Precisamente en relación con esta última observación, el planteamiento de la naturaleza del trabajo cognitivo nos permite dar un paso más allá, pasando a preguntarnos por los tipos de recursos e imputs "cognitivos" que hoy en día -con todas las ventajas que el tiempo pasado ofrece a la reflexión actual, recordemos que la corriente del capitalismo cognitivo tenía ante sí un panorama radicalmente diferente- están siendo utilizados provechosamente por la industria capitalista. Una simple mirada a la superficie fenomenológica nos permite hablar, grosso modo, de tres grandes grupos: por un lado, la fuerza de trabajo perteneciente al supuesto sector "creativo", aquella que se dedica al desarrollo y creación de software y/o tecnología que permita una mayor eficiencia, por tanto, una disminución de costes; por otro, trabajos que podríamos tildar de "creativos", pero que no suponen una novedad significativa para la estructura de acumulación del capital (aquí hablamos, muy en general, de profesiones liberales como abogacía, periodismo, enseñanza, etc.). Por último, habría de mencionarse un recurso en creciente disputa por su influencia para la valorización: la masa de interacciones en redes sociales y diferentes aplicaciones, que conforman el big data que se disputan gobiernos y empresas. Incluso cuando ni siquiera el último grupo mencionado puede ser considerado como "trabajo", estas diferentes realidades se entremezclan en los significados inducidos por los teóricos del capitalismo cognitivo, haciendo del desorden una constante de su argumentación.

La segunda de las cuestiones que plantea Caffentzis ${ }^{32}$ está relacionada con la inconmensurabilidad del trabajo cognitivo, que Vercellone presenta como el "ámbito de lo de fuera de medida" ${ }^{33}$. En este caso, basta un poco de historia y algún recordatorio de las bases fundamentales del marxismo para aclarar que dicha afirmación no tiene excesivo recorrido. Se trata de una afirmación sencillamente falsa: el conocimiento vivo que se valoriza a través de los derechos de propiedad intelectual es un proceso que sí se mide. Por no ir demasiado lejos, los límites de realización de un proyecto determinado (deadlines, bien conocidos en el mundo académico) son buena muestra de ello.

Adicionalmente, la afirmación de estos teóricos conduce a una reflexión más general asociada con la conmensurabilidad del valor, pues, según el marxista estadounidense, las proclamaciones en contra de la capacidad de medir algo suelen tener que ver con la falta de instrumentos $y / o$ conceptos para medir un objeto $y / o$ campo determinado. Este ha sido un problema recurrente a lo largo de la historia, que ha generado enormes desafíos a los que, no obstante, se han ofrecido soluciones $^{34}$. En definitiva, que algo no se pueda medir en un momento determinado, no quiere decir que no sea posible hacerlo, sino que quizás (todavía) no se ha encontrado la forma.

En tercer lugar y por último, también en relación con lo anterior, la afirmación de Vercellone\&Cía de que la

\section{Cf., ibidem}

33 C. Vercellone, "Crisis del valor...", op. cit., p. 221.

34 Un ejemplo puede ser la definición de los diversos tipos de infinito según el matemático G. Cantor. conversión de la interacción y la cooperación cognitiva, como uno de los factores principales de la valorización, hace entrar en contradicción el principio fundamental de la ley del valor basada en el tiempo de trabajo, es rotundamente falsa. Al menos, tal como la formulan. Vercellone afirma que, bajo el nuevo régimen de acumulación, el valor creado por el trabajo no puede ser medido "a partir del tiempo de trabajo directamente dedicado a su producción" 35 . En sentido estricto, esto no es algo exclusivo del capitalismo contemporáneo, sino que se trata de una peculiaridad inherente al sistema capitalista. Como bien enseñan los textos del recientemente fallecido $\mathrm{M}$. Postone $^{36}$, el tiempo de trabajo socialmente necesario no se determina con el tiempo cronometrado durante la producción de un objeto determinado por parte de un capitalista individual, sino que conforma un estándar social de productividad que conforma una determinada dinámica histórica. De hecho ${ }^{37}$, la dificultad de la medición del valor fue ya una preocupación de los teóricos del siglo XIX. Ya entonces, Marx se afanó en poner de manifiesto que el valor no era una magnitud que se pueda medir aisladamente respecto de sus circunstancias determinantes (como pudiera ser el caso de la medición del peso), sino que se trata de una forma social, y por tanto, dependiente de múltiples circunstancias ${ }^{38}$.

No pueden negarse -rompiendo una lanza a favor de Vercellone et al.- los cambios que ha habido en los últimos años en torno a la organización y medición de la calidad en el trabajo, así como a los nuevos malestares que se asocian a los nuevos fetiches de la "flexibilidad", la "resiliencia" y el "emprendimiento", entre otros. Pero desde luego, no se puede afirmar que estas hayan afectado a los mecanismos de acumulación del capital: la creciente financiarización y el uso de la política monetaria como incentivo a la producción que mencionan estos autores han de ser explicados por un conjunto de elementos que vayan más allá de la irrupción de las nuevas tecnologías y sus formas de trabajo asociadas. Cuanto menos, habría que empezar asumiendo el estudio de todas las otras ramas de la producción, que tal vez sean menos novedosas, pero igualmente decisivas.

35 C. Vercellone, "From formal...", op. cit., p. 30, en G. Caffentzis, "A critique...", op. cit., p. 112.

36 Cf. M. Postone, Tiempo, trabajo y dominación social. Madrid, Marcial Pons, 2006. Para una crítica de su punto de vista, cf. R. Kurz, "Die Substanz des Kapitals. Abstrakte Arbeit als gesellschaftliche Realmetaphysik und die absolute innere Schranke der Verwertung". Primera parte: "Die negative historisch-gesellschaftliche Qualität der Abstraktion "Arbeit»". En EXIT!. Krise und Kritik der Warengesellschaft, 1, pp. 44-129. Bad Honnef, Horlemann, 2004.

37 Cf. G. Caffentzis, "Immesurable value?...", op. cit., pp. 101-102

38 En este sentido, conviene recordar uno de los comentarios que Marx lanzaba a un recensionista de El Capital de su época. El autor alemán comenta que su obra magna comienza por la mercancía por ser esta una forma social simple -que no una sustancia o magnitud aisladaen la que se representa el valor. Marx dice lo siguiente: "De prime abord [de entrada, CNR], yo no parto de "conceptos", tampoco del "concepto de valor" y por tanto no tengo que "clasificar" este en modo alguno. De lo que yo parto es la manera social más simple en que el producto de trabajo se representa en la sociedad actual, y este es "la mercancía". Es esta la que analizo, y ciertamente, en primer lugar, en la manera en que aparece [erscheint]" (Marx, MEW 19, 368-369, cf. C. Ramas, Fetiche y mistificación capitalistas. La crítica de la economía política de Marx, Madrid, Siglo XXI, 2018, p. 82). 
Sea como fuere, independientemente de las deficiencias argumentativas de estos autores, creemos que ha quedado suficientemente demostrado que la confusión presente en su terminología resulta peligrosa, porque da a entender mucho más de lo que en realidad afirma. Hay dos asociaciones que, si bien solo se pueden inferir de manera indirecta de sus escritos, son particularmente alarmantes.

La primera de ellas es la que tiene que ver con la invisibilización de las condiciones que han permitido el surgimiento de los elementos asociados a las industrias y empleos cognitivos, así como el papel que cumplen dentro del conjunto de la estructura capitalista. Ya se ha explicado aquí a nivel conceptual de qué manera el proceso de valorización es un proceso que apela al conjunto de la clase capitalista, o sea, de qué modo, por decirlo abruptamente mediante una imagen, Google depende del trabajo de las maquiladoras. No obstante, es conveniente insistir en que la fijación de estos autores en la novedad de las empresas tecnológicas, así como en las consecuencias de su implementación para la acumulación, puede hacernos olvidar que i) ha sido una determinada correlación de fuerzas y conjunto de factores el que ha permitido el desarrollo y distribución de nuevas tecnologías y sus industrias asociadas, y que ii) su uso y extensión está relacionado con el mantenimiento y reiteración de las primeras. Por ello, antes de otorgar un poder transformador a un ámbito y/o fenómeno surgido de la matriz del desarrollo capitalista, hemos de preguntarnos hasta qué punto este está comprometido con su perpetuación para su sola existencia. ¿Resulta imaginable un mundo en el que el acceso a dispositivos tecnológicos sea tan sencilla como actualmente si se comienza a restituir un pago justo por las materias primas utilizadas en su construcción? ¿Es sostenible la acumulación sin la prestación del trabajo de cuidados no retribuido? ¿Estamos seguros de que la gratuidad de multitud de servicios en diversas webs y plataformas no depende de la cesión sin restricción de nuestros datos para su propio beneficio? ¿Realmente podemos considerar que nuestras interacciones virtuales suponen un nuevo modo de valorización, que abre una nueva fase en la acumulación del capitalismo? ¿Acaso los réditos provenientes de la gran mayoría de los mismos no están directamente relacionados y son dependientes de otras partes de la industria, siendo como es el caso que la mayoría de los beneficios de las plataformas de Internet más importantes -y cuya cotización en bolsa es cuanto menos significativaprovenientes de la publicidad? Las preguntas están tal vez algo desordenadas, pero son todas ellas, sin duda, pertinentes. En el campo de la economía política, donde todos los elementos están en mutua interrelación, la abstracción y aislamiento de sus diferentes fenómenos es, como poco, arriesgarse a errar.

La segunda de las asociaciones que hay que evitar a toda costa es la asociada al antiguo mito de la cornucopia. Con esta imagen nos referimos de manera algo impropia al deseo, de aparición intermitente a lo largo del desarrollo del capitalismo, de que el desarrollo tecnológico nos permita vivir en una sociedad de la abundancia, donde los bienes materiales sean suministrados sin esfuerzo alguno. La discusión sobre la posibilidad efectiva de que tal deseo pueda llevarse a cabo se ve hoy representado en los discursos de teóricos como Paul Mason y Jeremy Rifkin (y, con restricciones, también en la posición, mucho mejor fundamentada, de Nick Srnicek y Alex Williams) $)^{39}$.

Por lo que toca a los teóricos del capitalismo cognitivo, ha de admitirse que no se puede afirmar que en sus textos se defienda abiertamente esta argumentación. No obstante, hay una serie de factores que conducen a la impresión de que, en cierto modo, esta supone un supuesto implícito de su posición general. Ya la hemos comentado. Hablamos aquí de su constatación de que los factores cognitivos son un factor fundamental en la acumulación capitalista, afirmación aducida sin exponer simultáneamente de manera clara la multitud de condicionantes que lo hacen posible. Dicha aseveración se acompaña, además, de su insistente confianza en la capacidad disruptora de los factores cognitivos respecto de la ley de la plusvalía. En otras palabras: se afirma, con cierta rotundidad, la capacidad emancipatoria de ciertos elementos sin poner de manifiesto las cadenas que aseguran su dependencia a la reiteración de las relaciones de poder existentes. Aunque la relación entre los factores cognitivos y las relaciones de poder es contingente (como todo lo mundano), no hacerla explícita implica asumir tácitamente que los mencionados elementos poseen un potencial emancipatorio de carácter incondicionado y/o irrestricto. Cuanto menos, no se evita la amenaza de realizar tal interpretación. Para soslayar tal posibilidad, resulta útil poner de manifiesto el defecto argumentativo de la posición cornucopista, considerada más en general.

Expresado de manera abstracta y simplificada al extremo de la caricatura, el argumento cornucopista expresa que las máquinas pueden sustituir todo esfuerzo laboral, haciendo posible que la humanidad se encamine a un futuro sin esfuerzo. So far, so good, no obstante, este deseo ha de llevarse a cabo en el sistema capitalista, donde la introducción de maquinaria disminuye necesariamente la masa de plusvalor global, aquella de la que la clase capitalista en su conjunto obtiene sus beneficios ${ }^{40}$. La maquinaria, en general, la tecnología (capital constante), si bien ayuda a mejorar la eficiencia del proceso de producción de un bien determinado (permitiendo que una mayor cantidad de mercancías se produzca en menos tiempo) hace disminuir la fuerza de trabajo necesaria (capital variable) que, recordemos, es sustancia de valor ${ }^{41}$, única fuente del plusvalor y en último término, también de la ganancia. En el proceso de producción y valorización, el capital constante únicamente conserva valor, el capital variable, lo crea. El capitalismo ha de moverse, por tanto, entre la necesidad de mejorar la eficiencia de sus procesos de producción

\footnotetext{
39 Cf. J. Rifkin, The Zero Marginal Cost Society: The Internet of Things, The Collaborative Commons, and the Eclipse of Capitalism, Nueva York, Palgrave MacMillan, 2014; P. Mason, Postcapitalism. A Guide to our Future, Londres, Penguin Books, 2016, y A. Williams y N. Srnicek, Inventar el futuro. Postcapitalismo y un mundo sin trabajo, Barcelona, Malpaso, 2017.

40 Cf. G. Caffentzis, "The end of work...", op. cit., p. 73.

${ }^{41}$ Cf. R. Kurz, op. cit., pp. 48-53.
} 
al tiempo que de explotar a la mayor parte de trabajadores posible y, siendo así, la mejora tecnológica puede llevar a la pérdida de beneficios. Tal como se ha visto al comentar el fragmento de la estructura trinitaria, el capitalismo requiere, necesariamente, de la materia que conforma el interés, el beneficio y la renta, una materia que únicamente puede ser creada por la masa de trabajadores. Siendo así, su eliminación conduciría, de manera necesaria, a la destrucción del capitalismo. Un sistema, el capitalista, que no lo olvidemos, es uno y el mismo que ha hecho posible el desarrollo de cierta tecnología. Resulta claro, entonces, que hemos de ser cautelosos cuando el capitalismo ofrece un peldaño desde el que es posible observar un futuro de liberación: lo más probable es que dicho impulso venga acompañado de la correa que impide dejarlo definitivamente atrás.

\section{Mapa (mínimo) para la constitución de un análisis del capitalismo contemporáneo}

El estudio del capitalismo cognitivo que hemos llevado a cabo en estas líneas servía al objetivo fundamental de identificar sus principales deficiencias. Con ello, pretendíamos sentar las bases para un análisis más adecuado del capitalismo contemporáneo, desde el firme convencimiento de que el conocimiento de las aporías del pasado es el único modo de no volver a repetirlas. A la vista de lo analizado, y presuponiendo las ventajas que implica ser testigos del despliegue concreto de muchos de los factores que anteriormente no eran más que indicios, es el momento de indicar brevemente qué elementos ha de incluir un análisis crítico de la actual fase de la acumulación capitalista atenta a las novedades tecnológicas.

En primer lugar, creemos que ha de analizarse en qué tipos de empresas se está materializando la cacareada transformación del capitalismo, y en qué medida son dependientes de la configuración trabajo/energía que se supondría habrían de dejar atrás. Como sabemos por Srnicek $^{42}$, el modelo es el de la plataforma digital, cuya novedad frente al antiguo modelo de negocio (basado en la oferta de bienes y servicios) consiste en conectar a dos o más grupos. Su tipología es amplia, y abarca desde las plataformas publicitarias (Google, Facebook), de gestión (Uber) o industriales. La ventaja competencial que está provocando su éxito es el acceso a y gestión de la inmensa cantidad de datos de los usuarios que interactúan en ellas, lo que está inclinando a ciertos teóricos a considerar los datos como una nueva forma de riqueza. Un análisis riguroso habría de estudiar dos cuestiones. En primer lugar, a) el impacto económico de los datos generados por los usuarios y su correlación con el resto de la estructura capitalista. Si, como parece, la monetización del big data pasa, bien por servir de fundamento a la creación de publicidad personalizada, bien como fundamento para la reducción de costes, habría que po-

\footnotetext{
42 Cf. N. Srnicek, Platform Capitalism, Londres, Verso Books, 2017 (En adelante Platform...). (Hay traducción al castellano disponible: Capitalismo de plataformas, Buenos Aires, Caja Negra, 2018), de este mismo autor, cf. asimismo "Platform Monopolies and the Political Economy of AI", en J. McDonnell (ed.), Economics for the many, Londres, Verso, 2018.
}

ner en tela de juicio la sostenibilidad e interdependencia respecto de otras industrias de este modelo de negocio. Asimismo, b) ha de cuestionarse el contexto de surgimiento de este tipo de empresas, en un contexto de ínfimos tipos de interés y extrema financiarización de la economía, que puede llamar a los poseedores de dinero a invertir en proyectos de este tipo ${ }^{43}$.

En segundo lugar, y en correlación con el anterior punto, es necesario analizar la posible influencia del big data en el conjunto de la economía. Aunque ya se ha mostrado que en ningún caso pueden ser considerados meros sustitutos del dinero ${ }^{44}$, es importante observar de qué manera son capaces de incidir en las dinámicas de competencia mercantiles, lo que sí podría tener importantes consecuencias económicas. En correlación con el big data, es igualmente importante atender al instrumento de gestión de los mismos, el algoritmo. Componente fundamental del software, este se define como el conjunto de pasos estructurados para procesar una serie de instrucciones o datos para producir un resultado determinado $^{45}$. Lejos de poseer un carácter neutro o meramente instrumental, los algoritmos definen de qué modos se abordan ciertos problemas y, de hecho, su propia configuración como algoritmo define de antemano qué elementos configuran una situación determinada, algo con evidentes consecuencias éticas, filosóficas y epistemológicas que no podemos dejar de lado. Ya se ha insistido en la capacidad de los algoritmos en la perpetuación de ciertos estereotipos ligados al género $\mathrm{y}$, asimismo, uno de los últimos informes del relator especial de la ONU para los asuntos de extrema pobreza y derechos humanos alertaba de las graves amenazas para la autonomía y libertad que estaban teniendo el proceso de digitalización y automatización -léase conversión algorítmicade los procesos de gestión de protección social ${ }^{46}$.

En tercer lugar, es necesario comprender las transformaciones del trabajo que están teniendo lugar con este nuevo tipo de economía. Sin duda, es necesario seguir profundizando en el estudio, ya enormemente avanzado, de lo que significan los procesos de flexibilización de la fuerza de trabajo y la progresiva desaparición del salario en favor del pago por encargo y/o proyecto, así como sus consecuencias para la subjetividad de los trabajadores. Este estudio ha de realizarse desde una perspectiva global que comprenda la diferencia-

Cf. N. Srnicek, Platform..., op. cit., pp. 16-45 (versión .epub).

44 V. E. Morozov, "Digital Socialism? The Calculation Debate in the Age of Big Data", en New Left Review, 116-117, 2019, pp. 33-67.

45 Cf. R. Kitchin, "Thinking critically about and researching algorithms", en Information, Comunication \& Society, cf. 20, nº1, 2017, pp. 14-29, aquí p. 14. DOI: [http://dx.doi.org/10.1080/13691 18X.2016.1154087] La obra de este autor es especialmente valiosa para acercarse a esta cuestión.

46 La importancia de los algoritmos en la perpetuación de los sesgos de género y las posibilidades para su transfromación se ha estudiado en T. Bolukbasi, K.-W. Chang, J. Zou, V. Saligrama, A. Kalai, "Man is to Computer Programmer as Woman is to Homemaker? Debiasing Word Embeddings", en D. D. Lee, U. von Luxburg, R. Garnett, M. Sugiyama, I. Guyon (eds.), Proceedings of the 30th International Conference on Neural Information Processing Systems (NIPS'16), EE. UU, Curran Associates Inc., 2016, pp. 4356-4364. La referencia del informe de la ONU que hemos mencionado es A/74/48037, correspondiente a la sesión número 74 , con fecha del 11 de octubre de 2019. 
ción geográfica de los procesos de precarización y su correlación con la migración. Es igualmente crucial atender a las categorías tanto de empleo remunerado por el mercado, como al trabajo de reproducción y cuidados que, bien se realiza de manera gratuita, bien se da en condiciones de trabajo irregulares, afectando a las personas según su posición en la jerarquía de las vidas que merecen (en terminología butleriana) "ser 1loradas", en cuya cúspide se encuentra el sujeto BBVAh (blanco-burgués-varón-adulto-heterosexual) ${ }^{47}$.

Por lo que respecta a los aspectos más propiamente tecnológicos, se ha de añadir igualmente el estudio de los impactos que han supuesto el desarrollo de la máquina de Turing y la tecnología de la computación en el ámbito del disciplinamiento de los trabajadores y la organización de su trabajo. Como ejemplo, puede servir el cálculo de rutas por medio de algoritmos que ya se ha implementado - si bien con carácter no coercitivo- en algunas empresas de transporte. En este grupo de asuntos no debe olvidarse en ningún caso el trabajo de reproducción, que también es susceptible de transformaciones a causa de la tecnología.

Asimismo, es necesario, desde un punto de vista más general y global, comprender los cambios sociales y culturales que han provocado la irrupción de las tecnologías de la comunicación en nuestras sociedades, así como los espacios que habitamos. Este es un asunto que lleva tiempo siendo analizado por diversos autores. Habiendo mostrado sus luces y sombras, la tecnología parece un instrumento de doble filo que muestra sus aspectos más o menos virtuosos según el uso dado ${ }^{48}$.

Por último, y en ningún caso menos importante, resulta necesario comprender de manera rigurosa las condiciones y consecuencias materiales que tiene la implementación de las nuevas tecnologías y, más en general, el propio sostenimiento del statu quo capitalista. Si bien es cierto

\footnotetext{
47 Por lo que respecta a la flexibilización de la fuerza de trabajo, puede resultar útil el trabajo de $\mathrm{S}$. Bologna, Crisis de la clase media y posfordismo, Madrid, Akal, 2006, así como C. Morini en Por amor o a la fuerza. Feminización del trabajo y biopolítica del cuerpo, Madrid, Traficantes de Sueños, 2014. Asimismo, el trabajo de cuidados en el contexto global del neoliberalismo ha sido estudiado en S. López Gil y A. Pérez Orozco, Desigualdades a flor de piel: cadenas globales de cuidados. Concreciones en el empleo de hogar y articulaciones políticas, ONU Mujeres, 2011. Una perspectiva especialmente útil a las transformaciones tecnológicas y su impacto se encuentra en U. Huws, Labor in the Global Digital Economy, Nueva York, Monthly Review Group, 2014, mientras que el análisis de los posibles impactos del desarrollo de la computación en la organización del trabajo se encuentra en G. Caffentzis, "Las máquinas de Turing, la mano de obra cualificada y la teoría laboral del valor", en F. Sierra Caballero, op. cit., pp. 163-178. Una pequeña reflexión sobre su impacto en las tareas reproductivas se encuentra en H. Hester y N. Srnicek, The Age of Perplexity: Rethinking the World we Knew. The Crisis of Social Reproduction and the End of Work, Madrid, BBVA, OpenMind, Penguin Random House Grupo Editorial, 2017. Por último, el análisis de la transformación de la subjetividad que está teniendo lugar por las transformaciones que se van a considerar en estas líneas pueden ser considerados, entre otros, en J. Moruno, No tengo tiempo, Madrid, Akal, 2018, así como en N. Puertas, Al menos tienes trabajo, Valencia, Antipersona, 2019.

48 El gran referente teórico a este respecto es nuevamente E. Morozov, siendo especialmente pertinente su texto To save everything, click here. The folly of technological solutionism, Nueva York, Public Affairs, 2013. Cf., asimismo su texto "The taming of Tech criticism", en The Baffler, 27, 2015, disponible en la siguiente URL: [https:// thebaffler.com/salvos/taming-tech-criticism].
}

que el desarrollo tecnológico podría mejorar la eficiencia de los procesos de distribución de recursos, abriendo la puerta a la disminución de generación de residuos, no debe olvidarse que una de las tesis fundamentales que ha venido defendiendo el ecologismo crítico ha sido la necesidad de decrecer, reduciendo considerablemente nuestra actividad económica. Asimismo, se ha cuestionado fuertemente la posibilidad de existencia de un "capitalismo verde", disminuyendo pues las expectativas en torno a las supuestas promesas de la tecnología. Las intervenciones a este respecto son múltiples, incluyendo, entre otras, la perspectiva crítica ecofeminista y marxista, e incluyen análisis situados de consideración crítica del presente, dedicadas al estudio de fenómenos como el neoextractivismo o la industria agroalimentaria ${ }^{49}$.

$$
* * *
$$

Alguien que hubiera leído de manera poco atenta este texto podría verse sorprendido por haber terminado su exposición argumentativa hablando de cuestiones en apariencia escasamente relacionadas con el desarrollo tecnológico. No obstante, creemos que la constatación de datos que hemos realizado al comienzo de este artículo indica con claridad que la perspectiva que hemos asumido en estas líneas es la correcta. Ahí vimos cómo los indicadores tanto de obtención de beneficios como de capitalización en bolsa parecían favorecer, de igual modo, a empresas que consideramos símbolos del capitalismo más clásico (energéticas) y a los nuevos gallos del corral, que son los gigantes tecnológicos asociados a Silicon Valley. Nuestra intención ha sido hacer ver que si aparecen juntas es, quizá, porque dependen recíprocamente más de lo que nuestra propia imaginería social, que insiste en virtualidades y conexiones inmediatas, quiere reconocer. Nuestros análisis, por tanto, sin dejar de atender a las novedades que las empresas tecnológicas han introducido, no puede dejar de reconocer la influencia e importancia de los actores consolidados.

Se ha examinado cómo la novedad ofuscó la mirada de los teóricos del capitalismo cognitivo, que imaginaron posibilidades emancipatorias para elementos que en realidad, no poseían lo que parecían ofrecer. Este pequeño análisis, identificando sus desaciertos, es, aunque parezca contraintuivo, el mejor homenaje que podemos realizar a su contribución. Dado que no repetiremos sus errores, su esfuerzo ya no habrá sido en vano, y su labor conformará el secreto hilo rojo de todo análisis futuro. A los teóricos del capitalismo cognitivo solo resta agradecerles su labor. Todo lo demás, es una tarea a realizar que ya ha dado comienzo.

\footnotetext{
Desde el lado del marxismo debe nombrarse la intervención respecto de la lectura de Marx de K. Saito, Karl Marx's Ecosocialism: Capital, Nature, and the Unfinished Critique of Political Economy, Nueva York, Monthly Review Press, 2017, y desde el ecofeminismo, el clásico de M. Mies y V. Shiva, Ecofeminism, Londres, Zed Books, 2014, así como los textos dedicados a los problemas agroalimentarios de la última autora citada. Otras voces reconocidas son las de Yayo Herrero y Enrique Leff. Respecto del extraccionismo, es iluminador el texto de E. Gudynas, "Extracciones, extractivismos y extrahecciones. Un marco conceptual sobre la apropiación de recursos naturales", Observatorio del desarrollo, 18, 2013, pp. 1-18.
} 


\section{Bibliografía}

Bhattacharya, T., "Explaining gender violence in the neoliberal era", International Socialist Review, 91, disponible en la siguiente URL: [https://isreview.org/issue/91/explaining-gender-violence-neoliberal-era].

Blondeau, O., Dyer Whiteford, N., Vercellone, C., Kyrou, A., Corsani, A., Rullani, E., Moulier-Boutang, Y., y Lazzarato, M., Capitalismo cognitivo. Propiedad intelectual y creación colectiva, Madrid, Traficantes de Sueños, 2004.

Bologna, S., Crisis de la clase media y posfordismo, Madrid, Akal, 2006.

Bolukbasi, T., Chang, K.-W., Zou, J., Saligrama, V., Kalai, A., "Man is to Computer Programmer as Woman is to Homemaker? Debiasing Word Embeddings”, en Lee, D.D., von Luxburg, U., Garnett, R., Sugiyama, M., Guyon, I. (eds.), Proceedings of the 30th International Conference on Neural Information Processing Systems (NIPS'16), EE. UU, Curran Associates Inc., 2016, pp. 4356-4364.

Caffentzis, G., "Immesurable Value? An Essay on Marx's Legacy”, The Commoner, 10, 2005, pp. 87-114.

-, In letters of Blood and Fire. Work, machines and the Crisis of Capitalism, Oakland, PM Press, 2013.

Federici, S., El patriarcado del salario. Críticas feministas al marxismo, Madrid, Traficantes de Sueños, 2018.

Fumagalli, A., Lucarelli, S., Marazzi, C., Mezzadra, S., Negri, A., y Vercellone, C., La gran crisis de la economía global. Mercados financieros, luchas sociales y nuevos escenarios políticos, Madrid, Traficantes de Sueños, 2009.

Gudynas, E., "Extracciones, extractivismos y extrahecciones. Un marco conceptual sobre la apropiación de recursos naturales", Observatorio del desarrollo, 18, febrero, 2013, pp. 1-18.

Huws, U., Labor in the Global Digital Economy, Nueva York, Monthly Review Group, 2014.

Kitchin, R., "Thinking critically about and researching algorithms", en Information, Comunication \& Society, 20, 1, 2017, pp. 1429. DOI: [http://dx.doi.org/10.1080/1369118X.2016.1154087].

Kurz, R., "Die Substanz des Kapitals. Abstrakte Arbeit als gesellschaftliche Realmetaphysik und die absolute innere Schranke der Verwertung". Primera parte: "Die negative historisch-gesellschaftliche Qualität der Abstraktion «Arbeit»". En EXIT!. Krise und Kritik der Warengesellschaft, 1, pp. 44-129. Bad Honnef, Horlemann, 2004.

López Gil, S. y Pérez Orozco, A., Desigualdades a flor de piel: cadenas globales de cuidados. Concreciones en el empleo de hogar y articulaciones politicas, ONU Mujeres, 2011.

Marx, K., Das Kapital. Kritik der Politischen Ökonomie. Tercer tomo, en Karl Marx-Friedrich Engels Werke [MEW] B. 25, Berlín, Dietz, 2008 [1894]. (Traducción al castellano utilizada y citada: (2009) [1981]. El Capital. Crítica de la economía política, Tomo III, Traducción de Pedro Scaron, Madrid, Siglo XXI Editores).

Mason, P., Postcapitalism. A Guide to our Future, Londres, Penguin Books, 2016.

Mies, M., y Shiva, V., Ecofeminism, Londres, Zed Books, 2014.

Morini, C., Por amor o a la fuerza. Feminización del trabajo y biopolítica del cuerpo, Madrid, Traficantes de Sueños, 2014.

Morozov, E., To save everything, click here. The folly of technological solutionism, Nueva York, Public Affairs, 2013.

-, "The taming of Tech criticism", The Baffler, 27, Marzo 2015, disponible en la siguiente URL: [https://thebaffler.com/salvos/ taming-tech-criticism].

-, "Digital Socialism? The Calculation Debate in the Age of Big Data", en New Left Review, 116-117, 2019, pp. 33-67.

Moruno, J., No tengo tiempo, Madrid, Akal, 2018.

Moulier-Boutang, Y., Cognitive Capitalism, Cambridge, Polity Press, 2011.

Negri, T., Marx más allá de Marx, Madrid, Akal, 2011.

-, y Hardt, M., Imperio, Barcelona, Paidós, 2002

-, Multitud, Barcelona, Debate, 2004

-, Commonwealth, Madrid, Akal, 2011.

-, y Lazzarato, M., “Trabajo inmaterial y subjetividad”, Publicado originalmente en Futur Anteriéur, 6, 1991. Disponible en la siguiente URL [http://www.rebelion.org/docs/121986.pdf])

Postone, M., Tiempo, trabajo y dominación social, Madrid, Marcial Pons, 2006.

Puertas, N., Al menos tienes trabajo, Valencia, Antipersona, 2019.

Ramas, C., Fetiche y mistificación capitalistas. La crítica de la economía política de Marx, Madrid, Siglo XXI, 2018.

Rifkin, J., The Zero Marginal Cost Society: The Internet of Things, The Collaborative Commons, and the Eclipse of Capitalism, Nueva York, Palgrave MacMillan, 2014.

Saito, K., Karl Marx's Ecosocialism: Capital, Nature, and the Unfinished Critique of Political Economy, Nueva York, Monthly Review Press, 2017.

Sierra Caballero, F., (ed.), Teoría del valor, comunicación, territorio, Madrid, Akal, 2019.

Srnicek, N., Platform Capitalism, Londres, Verso Books, 2017 (Hay traducción al castellano disponible: Capitalismo de plataformas, Buenos Aires, Caja Negra, 2018)

-, "Platform Monopolies and the Political Economy of AI", McDonnell, J., (ed.), Economics for the many, Londres, Verso, 2018.

-, y Hester, H., The Age of Perplexity: Rethinking the World we Knew. The Crisis of Social Reproduction and the End of Work, Madrid, BBVA, OpenMind, Penguin Random House Grupo Editorial, 2017.

-, y Williams, A., Inventar el futuro. Postcapitalismo y un mundo sin trabajo, Barcelona, Malpaso, 2017.

Vercellone, C., "From formal subsumption to general intellect: elements for a Marxist reading of the thesis of cognitive capitalism", Historical Materialism, 17, 2007, pp. 13-36 DOI: [https://doi.org/10.1163/156920607X171681]. 